\title{
Fiberwise stable bundles on elliptic threefolds with relative Picard number one
}

\author{
Andrei CĂLDĂRARU \\ Mathematics Department, University of Massachusetts, Amherst, MA 01003, USA \\ E-mail: andreic@math.umass.edu
}

(Reçu le 29 November 2001, accepté le 7 January 2002)

\begin{abstract}
We show that fiberwise stable vector bundles are preserved by relative Fourier-Mukai transforms between elliptic threefolds with relative Picard number one. Using these bundles we define new invariants of elliptic fibrations, and we relate the invariants of a space with those of a relative moduli space of stable sheaves on it. As a byproduct, we calculate the intersection form of a certain new example of an elliptic Calabi-Yau threefold. (C) 2002 Académie des sciences/Éditions scientifiques et médicales Elsevier SAS

Fibrés vectoriels relativement stables sur variétés elliptiques de dimension trois dont le numéro relatif de Picard est un

Résumé. Nous prouvons que les fibrés vectoriels relativement stables sont préservés par des transformées de Fourier-Mukai entre variétés elliptiques de dimension trois dont le numéro relatif de Picard est un. En utilisant ces fibrés nous définissons des nouveaux invariants de variétés elliptiques, et nous étudions la relation entre les invariants d'une variété et ceux d'un éspace relatif de modules des fibrés stables sur elle. Ces résultats nous permettent de calculer la forme d'intersection sur un certain nouvel exemple de variété de Calabi-Yau de dimension trois. (C) 2002 Académie des sciences/Éditions scientifiques et médicales Elsevier SAS
\end{abstract}

\section{Introduction}

The object of this note is to generalize to the case of elliptic threefolds with relative Picard number one classic results regarding stable vector bundles on elliptic curves and their Fourier-Mukai transforms. Several results of this type are known: partial results by Bridgeland ([3]) for arbitrary elliptic fibrations, and strong results by Bartocci, Bruzzo, Hernández-Ruipérez and Muñoz-Porras ([2]) for elliptic fibrations without reducible fibers. Our primary interest is in applying this theory to the study of elliptic Calabi-Yau threefolds, so we need to have results general enough to handle reducible curves of arithmetic genus 1 .

Stable bundles on an elliptic curve $E$, of rank $r$ and degree $d,(r, d)=1$, are well understood:

THEOREM (Atiyah [1]). - For every line bundle $\mathscr{L}$ of degree $d$ on E, there exists a unique stable vector bundle $V(r, \mathscr{L})$ of rank $r$ and determinant $\mathscr{L}$.

Note présentée par Jean-Pierre DEMAILLY

S1631-073X(00)002290-2/FLA

(c) 2002 Académie des sciences/Éditions scientifiques et médicales Elsevier SAS. Tous droits réservés. 


\section{Andrei CĂLDĂRARU}

THEOREM (Tu [8]). - A vector bundle $\mathscr{F}$ on $E$ of rank $r$ and degree $d$ is stable if and only if it is simple (i.e. $\operatorname{Hom}(\mathscr{F}, \mathscr{F})=\mathbf{C}$ ).

Let $a, b$ be coprime integers, with $a>0$, and let $M$ be the moduli space of stable bundles of rank $a$, degree $b$ on $E$ (which is a fine moduli space). Fix a universal sheaf $\mathscr{U}$ on $X \times M$, and let $c$ be the degree of the restriction $\left.\mathscr{U}\right|_{\{x\} \times M}$ for $x \in X$, which is independent of $x$. Let $p_{E}, p_{M}$ be the projections from $E \times M$ to $E, M$, respectively.

THEOREM (Bridgeland [3]). - Let $\mathscr{F}$ be a stable vector bundle on $M$, of slope $\mu(\mathscr{F}) \neq-c / a$. Then the Fourier-Mukai transform $\mathscr{G}$ of $\mathscr{F}, \mathscr{G}=\Phi_{M \rightarrow E}^{\mathscr{U}}(\mathscr{F})=\mathbf{R} p_{E, *}\left(p_{M}^{*}(\mathscr{F}) \otimes \mathscr{U}\right)$ is a stable vector bundle on $E$ of slope $\mu(\mathscr{G}) \neq b / a$, possibly shifted.

The stability of $\mathscr{F}$ is used in two ways in the proof of the above theorem. It is first used to conclude that $\mathscr{G}$ consists of a single vector bundle, possibly shifted. Then, since $\mathscr{F}$ is simple and simplicity is preserved by Fourier-Mukai transforms, $\mathscr{G}$ is simple, and thus stable by Tu's theorem.

\section{Fiberwise stable bundles}

Our goal is to replace the elliptic curve $E$ in the above results by an elliptic threefold $X / S$, with $X$ and $S$ smooth, thus allowing for some reducible fibers. In such generality, only partial results are known, mainly due to Bridgeland ([4]). Note that the theorems of Atiyah and Tu hold when $E$ is replaced by an irreducible curve of arithmetic genus 1, but fail if $E$ is reducible. We'll assume that the fibration $X / S$ has no multiple fibers, its relative Picard number $\rho(X / S)=\rho(X)-\rho(S)$ is equal to one, $\operatorname{Pic}(X)$ has no torsion, and the general singular fiber of $X / S$ is irreducible. These conditions are often satisfied by general members of families of elliptic Calabi-Yau threefolds ([7]). The assumption that $X$ and $S$ are smooth ensures that all the fibers of $X \rightarrow S$ are Cohen-Macaulay.

Given such an elliptic fibration $X / S$, and $r, d$ coprime integers with $r>0$, denote by $V_{X}(r, d)$ the class of vector bundles of rank $r$ on $X$, whose restriction to each fiber of $X / S$ is stable of degree $d$. Since we are dealing with possibly reducible fibers, we use Gieseker's definition of stability. In general, the definition of $V_{X}(r, d)$ depends on the choice of polarization in each fiber, but the assumption $\rho(X / S)=1$ implies that each fiber is polarized in a unique way (up to multiples) by the restriction of a polarization of $X$. From here on, we'll always assume the fibers polarized in this way. The case $r<0$ can also be included in the definition, by considering elements of $V_{X}(r, d)$ as objects in $\mathbf{D}_{\text {coh }}^{b}(X)$ which consist of a single sheaf, possibly shifted (if $\mathscr{E}$ is a $V(r, d)$, then $\mathscr{E}[1]$ is a $V(-r,-d)$ ).

PROPOSITION 1. - Elements of $V_{X}(r, d)$ differ by tensoring by pull-backs of line bundles in $\operatorname{Pic}(S)$ and by even shifts in the derived category.

Proof. - Follows from Atiyah's result on irreducible curves of genus 1 and Hartogs' theorem.

We want to study the behaviour of the $V(r, d)$-bundles under Fourier-Mukai transforms. Let $n$ be the smallest positive degree of a multisection of $X / S$ (alternatively, this is the smallest degree of a polarization of the fibers which is the restriction of a polarization from $X / S$ ). Consider integers $a>0$ and $b$ so that $(n a, b)=1$, and let $M / S$ be the relative moduli space of stable sheaves of rank $a$, degree $b$ on the fibers of $X / S$, in the sense of Simpson. The space $M$ has a natural map to $S$ which makes it into an elliptic fibration which satisfies all the conditions imposed on $X / S$. The fibration $M / S$ is a fine moduli space, and the extension by zero $\mathscr{U}$ to $X \times M$ of a universal sheaf on $X \times_{S} M$ induces a Fourier-Mukai transform $\Phi_{M \rightarrow X}^{\mathscr{U}}: \mathbf{D}_{\text {coh }}^{b}(M) \rightarrow \mathbf{D}_{\text {coh }}^{b}(X)([3],[4])$. Let $c$ be the degree of the restriction of $\mathscr{U}$ to a fiber $\{x\} \times M$ for $x \in X$ and $e=(b c-1) / a$. For $r$ and $d$ coprime define $V_{M}(r, d)$ in an analogous fashion to $V_{X}(r, d)$.

THEOREM 2. - If $\mathscr{F}$ is a $V_{M}(r, d)$, for $r, d$ coprime, $d / r \neq-c / a$, then the Fourier-Mukai transform $\mathscr{G}=\Phi_{M \rightarrow X}^{\mathscr{U}}(\mathscr{F})$ is a $V_{X}\left(r^{\prime}, d^{\prime}\right)$, where $\left(r^{\prime}, d^{\prime}\right)$ are given by

$$
\left(\begin{array}{l}
r^{\prime} \\
d^{\prime}
\end{array}\right)=\left(\begin{array}{ll}
c & a \\
e & b
\end{array}\right)\left(\begin{array}{l}
r \\
d
\end{array}\right) \text {. }
$$


Fiberwise stable bundles on elliptic threefolds

The relationship between $\left(r^{\prime}, d^{\prime}\right)$ and $(r, d)$ has been known previously ([3]); what is new is the fact that $\mathscr{G}$ is a $V_{X}\left(r^{\prime}, d^{\prime}\right)$. The proof of Theorem 2 follows the same lines as the proof of Bridgeland's theorem, and in order to conclude that $\mathscr{G}$ is a single, locally free sheaf on $X$ we need to have the restriction of $\mathscr{F}$ to every fiber of $M / S$ be stable. (This part of the proof goes through with almost no restrictions on $X / S$.) But although we are guaranteed that the restriction of $\mathscr{G}$ to all the fibers of $X / S$ is simple, we can no longer apply Tu's result to conclude that it is stable. Thus, for general fibrations, the situation is asymmetric (we need to start with a sheaf that is stable on all the fibers, and we end up with one that is only simple). The crucial observation in the case of relative Picard number one is the following:

Proposition 3. - Let E be a (possibly reducible) Cohen-Macaulay curve of arithmetic genus 1 , and let $\mathscr{E}$ be a locally free sheaf on $E$ whose determinant is either ample or anti-ample. Then $\mathscr{E}$ is simple if and only if it $\mathscr{E}$ is stable with respect to the polarization induced by $\operatorname{det} \mathscr{E}$ or $-\operatorname{det} \mathscr{E}$.

Proof. - One implication is trivial, so assume that $\mathscr{E}$ is simple, and that $\mathscr{L}=\operatorname{det} \mathscr{E}$ is ample. Then $\mathscr{L}=\mathscr{O}\left(\sum a_{i} P_{i}\right)$, with $a_{i}>0$ and $P_{i} \in E^{\text {smooth }}$. For any torsion-free sheaf $\mathscr{F}$ on $E$, let

$$
\mu_{\mathscr{L}}(\mathscr{F})=\frac{\chi(\mathscr{F})}{\sum a_{i} \operatorname{rk}_{P_{i}}(\mathscr{F})},
$$

where $\operatorname{rk}_{P_{i}}(\mathscr{F})=\operatorname{dim} \mathscr{F}_{P_{i}}$. The reduced Hilbert polynomial of $\mathscr{F}$, computed with respect to $\mathscr{L}$, is equal to $t+\mu_{\mathscr{L}}(\mathscr{F})$. Thus, to show that $\mathscr{E}$ is stable with respect to $\mathscr{L}$, we need to show that if $\mathscr{F}$ is a proper subsheaf of $\mathscr{E}$, then $\mu_{\mathscr{L}}(\mathscr{F})<\mu_{\mathscr{L}}(\mathscr{E})$. A straightforward computation shows that $\mu_{\mathscr{L}}(\mathscr{F} \otimes \mathscr{E} \vee)=$ $\mu_{\mathscr{L}}(\mathscr{F})-\mu_{\mathscr{L}}(\mathscr{E})$. If $\mathscr{F}$ is a subsheaf of $\mathscr{E}, \operatorname{Hom}(\mathscr{F}, \mathscr{E}) \neq 0$ and thus $H^{1}(E, \mathscr{F} \otimes \mathscr{E} \vee)=\operatorname{Ext}^{1}(\mathscr{E}, \mathscr{F})=$ $\operatorname{Hom}(\mathscr{F}, \mathscr{E}) \neq 0$ by Serre duality (which can be applied because $\mathscr{E}$ is locally free). If $\mu_{\mathscr{L}}(\mathscr{F}) \geq \mu_{\mathscr{L}}(\mathscr{E})$, then $\chi\left(\mathscr{F} \otimes \mathscr{E}^{\vee}\right) \geq 0$, so we conclude that $\operatorname{Hom}(\mathscr{E}, \mathscr{F})=H^{0}\left(E, \mathscr{F} \otimes \mathscr{E}^{\vee}\right) \neq 0$. Thus there is a non-zero map $\mathscr{E} \rightarrow \mathscr{F}$, which composed with the inclusion $\mathscr{F} \subset \mathscr{E}$ yields a non-trivial map $\mathscr{E} \rightarrow \mathscr{E}$, contradicting the assumption that $\mathscr{E}$ is simple. The case $\operatorname{det} \mathscr{E}$ anti-ample is treated in a similar way.

COROLLARY 4. - Let $\mathscr{E}$ be a locally free sheaf on $X$. Then its restriction to a fiber of $X / S$ is stable (with respect to the unique polarization of the fiber coming from $X$ ) if and only if it is simple.

Note that det $\mathscr{E}$ must restrict to a multiple of the polarization $\mathscr{O}_{X / S}(1)$ on each fiber, because $\rho(X / S)=$ 1. If this restriction is non-trivial, we can apply Proposition 1 If $\operatorname{det} \mathscr{E}$ is the trivial line bundle in each fiber, $\mathscr{E}(1)$ is stable in each fiber with respect to $\mathscr{O}_{X / S}(1)$, which implies that $\mathscr{E}$ is fiberwise stable.

\section{Invariants of elliptic fibrations}

We'd like to have invariants that enable us to compare $X$ and $M$. Define $P_{X}(r, d)$ to be the class of elements of $\mathbf{D}_{\mathrm{coh}}^{b}(S)$ that are of the form $\mathbf{R} \pi_{X, *} \mathscr{F}$ for $\mathscr{F}$ a $V_{X}(r, d)\left(\pi_{X}: X \rightarrow S\right.$ is the structural map of the fibration $X / S)$. Define $P_{M}(r, d)$ analogously.

The following two propositions are applications of Proposition 1 the projection formula, GrothendieckSerre duality and the fact that the dual of a $V(r, d)$ is a $V(r,-d)$, which follows from Proposition 3

Proposition 5. - For $d \neq 0$, elements of $P_{X}(r, d)$ are vector bundles (possibly shifted) which differ by tensoring by line bundles in $\operatorname{Pic}(S)$ and by even shifts in the derived category.

Proposition 6. $-P_{X}(r, d)=P_{X}(r,-d)^{\vee}[1]=P_{X}(-r, d)^{\vee}=P_{X}(-r,-d)[1]$, where $P_{X}(r,-d)^{\vee}[1]$ is obtained by dualizing and shifting every element of $P_{X}(r,-d)$, and $P_{X}(-r, d)^{\vee}, P_{X}(-r,-d)[1]$ are defined in a similar way.

THEOREM 7. - We have $P_{X}\left(r^{\prime}, d^{\prime}\right)=\mathbf{R} \pi_{M, *}\left(V_{M}(r, d) \otimes V_{M}(b, e)\right)$, where $a, b, c$, e and $r, d, r^{\prime}, d^{\prime}$ are as in Theorem [2] In particular, if $r=1, P_{X}(c+a d, e+b d)=P_{M}(b, e+b d)$.

Proof. - Let $\mathscr{F}$ be a $V_{M}(r, d)$, and let $\mathscr{G}=\Phi_{M \rightarrow X}^{\mathscr{U}}(\mathscr{F})=\mathbf{R} p_{X, *}\left(p_{M}^{*} \mathscr{F} \stackrel{\mathrm{L}}{\otimes} \mathscr{U}\right)$, where $p_{X}, p_{M}$ are the projections from $X \times M$ to $X$ and $M$, respectively. Then $\mathscr{G}$ is a $V_{X}\left(r^{\prime}, d^{\prime}\right), \mathbf{R} \pi_{X, *} \mathscr{G}=\mathbf{R} \pi_{M, *}(\mathscr{F} \otimes$ $\left.\mathbf{R} p_{M, *} \mathscr{U}\right)$, and all we need to show is that $\mathbf{R} p_{M, *} \mathscr{U}$ is a $V_{M}(b, e)$. But $\mathbf{R} p_{M, *} \mathscr{U}=\Phi_{X \rightarrow M}^{\mathscr{U}}\left(\mathscr{O}_{X}\right)$, so the 


\section{Andrei CĂLDĂRARU}

result follows from Theorem 2 once we show that $X$ is the relative moduli space of stable sheaves of rank $a$, degree $c$ on $M$, and $\mathscr{U}$ is a universal sheaf for this moduli problem. The proof of this fact (which is known for elliptic surfaces, [3]) will be presented elsewhere.

THEOREM 8. - If X/S also satisfies the property of being generic (see [7 for a definition), then $P_{X}(r, d)=P_{X}\left(r^{\prime}, d\right)$ for $r=r^{\prime} \bmod d$.

The proof of Theorem 8 is more involved, requiring the use of twisted derived categories ([6]), and will be included in a future paper. In the case of a single elliptic curve $E$, the proof does not rely on twisted sheaves, and we include a sketch here. If $\mathscr{F}$ is a $V_{E}(r, d)$, then its $\operatorname{transform} \mathscr{G}$ to $\mathrm{Jac}(E)$ is a $V_{\mathrm{Jac}(E)}(-d, r)$. On $\operatorname{Jac}(E)$ there is a naturally defined $\mathscr{O}_{\mathrm{Jac}(E)}(-1)$, and $\mathscr{G}(-1)$ is a $V_{\mathrm{Jac}(E)}(-d, r+d)$. Transforming $\mathscr{G}(-1)$ back to $E$ yields a $V_{E}(r+d, d)$, whose global sections can be shown to be the same as those of $\mathscr{F}$.

\section{An explicit calculation}

In 6 6 6.2.2] we studied an explicit generic elliptic Calabi-Yau threefold $X / \mathbf{P}^{2}$, constructed as the pfaffian of a certain $5 \times 5$ matrix of bihomogeneous forms on $\mathbf{P}^{2} \times \mathbf{P}^{4}$. We described all the minimal birational models of $X$, which consist of $X$, a flop $X^{+}$of $X$ which is contained in $\mathbf{P}^{4} \times \mathbf{P}^{5}$ and has no elliptic fibration structure, and a flop $X^{++}$of $X^{+}$contained in $\mathbf{P}^{5} \times \mathbf{P}^{2}$, which has an elliptic fibration structure given by the map $X^{++} \rightarrow \mathbf{P}^{2}$. There is no apparent relation between the original elliptic fibration structure on $X$ and the one on $X^{++}$. Since $X^{++}$is obtained from $X$ through a sequence of flops, $\mathbf{D}_{\text {coh }}^{b}\left(X^{++}\right) \cong \mathbf{D}_{\text {coh }}^{b}(X)([5])$.

On $X$ we have $n=5$; taking $a=1, b=2$ we obtain an elliptic Calabi-Yau threefold $M / \mathbf{P}^{2}$, with $\mathbf{D}_{\mathrm{coh}}^{b}(X) \cong \mathbf{D}_{\mathrm{coh}}^{b}(M)$. It can be shown that $V_{X}(1,5)$ and $V_{X}(3,5)$ are non-empty, and thus $V_{X}(r, 5)$ is non-empty for all $r$ relatively prime to 5. Comparing the invariants $P_{X}(r, 5)$ and $P_{M}(r, 5)$ for all $r$ relatively prime to 5 and using Grothendieck-Riemann-Roch, we compute $c_{2}(M)$ and the cubic form on $H^{2}(M, \mathbf{Z})$. These topological invariants can be computed for $X^{++}$, and they are the same as those of $M$.

Thus the two Calabi-Yau threefolds $X^{++}$and $M$ have $\mathbf{D}_{\text {coh }}^{b}\left(X^{++}\right) \cong \mathbf{D}_{\text {coh }}^{b}(M)$, and have the same $c_{2}$ and cubic form. In view of Wall's results ([9]), it seems natural to conjecture that $X^{++}$and $M$ are deformation equivalent. If they are non-isomorphic, this is likely to lead to a new counterexample to Torelli for Calabi-Yau threefolds (the equivalence between the derived categories of $X$ and $M$ induces an isomorphism between their polarized Hodge structures). In this counterexample, $X^{++}$and $M$ would be non-birational, since we can enumerate all the birational models of $X^{++}$and none of them is isomorphic to $M$. If $X^{++}$and $M$ are isomorphic, this would suggest that there is some deeper phenomenon behind this occurrence, which is worth investigating. It would also yield a new automorphism of the derived category of $X^{++}$, which is interesting to study in view of implications to Kontsevich's homological mirror symmetry conjecture.

Acknowledgements. I am grateful to Mark Gross for suggesting me to look at elliptic threefolds for counterexamples to Torelli, and to Sorin Popescu, Titus Teodorescu and Eyal Markman for numerous useful discussions.

\section{References}

[1] Atiyah, M. F., Vector bundles over an elliptic curve, Proc. London Math. Soc. (3) 71957 414-452

[2] Bartocci, C., Bruzzo, U., Hernández Ruipérez, D., Muñoz Porras, J., Relatively stable bundles over elliptic fibrations, preprint, alg-geom 0109123

[3] Bridgeland, T., Fourier-Mukai transforms for elliptic surfaces, J. reine angew. math. 498 (1998) 115-133

[4] Bridgeland, T., Maciocia, A., Fourier-Mukai transforms for K3 fibrations, preprint, alg-geom/9908022

[5] Bridgeland, T., Flops and derived categories, preprint, alg-geom/0009053

[6] Căldăraru, A., Derived Categories of Twisted Sheaves on Calabi-Yau Manifolds, Ph.D. thesis, Cornell University (2000)

[7] Căldăraru, A., Derived categories of twisted sheaves on elliptic threefolds, to appear in J. reine angew. math.

[8] Tu, L. W., Semistable bundles over an elliptic curve, Adv. Math. 98 (1993), no. 1, 1-26

[9] Wall, C. T. C., Classification problems in differential topology. V. On certain 6-manifolds, Invent. Math. 1 (1966), 355-374; corrigendum, ibid 21966306 